\title{
Photonic liquid crystal fibers with low-, medium-, and high refractive indices of the core
}

\author{
Sławomir Ertman ${ }^{* 1}$, Tomasz R. Woliński ${ }^{1}$, Ryszard Buczyński ${ }^{2}$, Dariusz Pysz ${ }^{3}$, \\ Edward Nowinowski-Kruszelnicki ${ }^{4}$, Roman Dąbrowski ${ }^{4}$ and Jan Wójcik $^{5}$ \\ ${ }^{1}$ Faculty of Physics, Warsaw University of Technology, Koszykowa 75, 00-662 Warszawa, Poland \\ ${ }^{2}$ University of Warsaw, ${ }^{3}$ Institute of Electronics Materials Technology, Warsaw, Poland \\ ${ }^{4}$ Military University of Technology, Warsaw ${ }^{5}$ Maria Curie-Skłodowska University, Lublin, Poland
}

Received September 24, 2010; accepted September 30, 2010; published September 30, 2010

\begin{abstract}
Photonic liquid crystal fibers (PLCFs) combine unique properties of microstructured optical fibers and liquid crystal fibers. In this paper we show that PLCFs can be divided into three classes with different guiding properties, depending on the relation between the refractive index of the core of the host fiber and refractive indices of the liquid crystal.
\end{abstract}

Photonic crystal fibers (PCFs) are attracting significant interest since they allow for remarkable control of guiding properties [1]. The propagation properties of PCFs can be dynamically tuned by filling their air-holes with various substances, and liquid crystals (LCs) are particularly interesting for this purpose since they exhibit very high electro-optic and thermo-optic effects.

Generally, in these fibers light can be guided by two different mechanisms: index-guiding (similar to the classical waveguide effect based on total internal reflection) and the photonic band-gap effect (Fig. 1). PBG propagation occurs when the effective (average) refractive index of the microstructured cladding is higher than the refractive index of the core, and in this case only selected wavelengths can be guided. So far most researchers have been using the PCFs made of silica glass, whose refractive index $(\sim 1.46)$ is lower than refractive indices of most liquid crystals, so PBG propagation was only possible. There have been a number of papers presenting thermal tuning of PBGs (i.e. [2-5]). Later also electric tuning of PBGs was obtained (i.e. [6, 7]). There have been also a number of works with more sophisticated tuning of PBGs in PLCFs, i.e. all-optical tuning in a PCF filled with a LC doped either with dyes [8] or with azobenzene [9], and frequency tunability of the PCF filled with dual frequency $[10,11]$ or nanoparticle-doped liquid crystals [12]. It is worth mentioning that together with PBG tuning also effective control of polarization properties was demonstrated [i.e. 13-14].

*E-mail: ertman@if.pw.edu.pl

Index guiding in PLCFs was observed for the first time in the Ref. [7], where thermal tuning of the guiding mechanism was obtained by using a special LC mixture with the ordinary refractive index lower (in a certain temperature range) than the refractive index of silica glass. Later on, low-loss index-guiding propagation was obtained in a PLCF based on a PCF made of high-index glass [15].
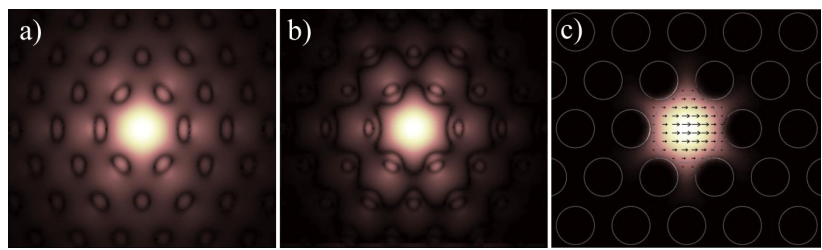

Fig. 1. Examples of modes profiles guided in PLCFs (PCFs) by the photonic band-gap phenomenon ( $\mathrm{a}, \mathrm{b})$ and index-guided mode (c).

It seems natural that the guiding properties of a PLCF strongly depend on the relation between the refractive index of the host fiber's core $\left(\mathrm{n}_{\mathrm{PCF}}\right)$ and both ordinary $\left(\mathrm{n}_{\mathrm{LCo}}\right)$ and extraordinary $\left(\mathrm{n}_{\mathrm{LCe}}\right)$ refractive indices of the liquid crystal. However, so far this issue has not been discussed. In this letter we divide PLCFs into three classes with a different relation between $\mathrm{n}_{\mathrm{PCF}}$ and $\mathrm{n}_{\mathrm{LCo}} / \mathrm{n}_{\mathrm{LCe}}$ and briefly discuss the main properties of each class.

a)

b)

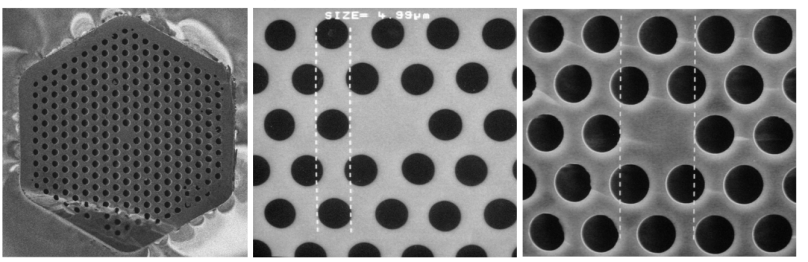

Fig. 2. Three types of the host PCFs discussed in this paper: a) MCSU 1023 made of silica glass - refr. index $\sim 1.45$; b) IEMT PCF-18 made of Schott F2 glass - refr. index $\sim 1.62$; c) IEMT PCF-14 made of PBG08 glass [16] - refr. index $\sim 1.95$ (hole diameters are $4.8 \mu \mathrm{m}, 5 \mu \mathrm{m}, 5.2 \mu \mathrm{m}$ and distances between holes are $6.5 \mu \mathrm{m}, 8.2 \mu \mathrm{m}, 7.6 \mu \mathrm{m}$ respectively) 


\section{Low index-core PLCFs}

As low index-core PLCFs we recognize the fibers based on solid-core PCFs made of glass, whose refractive index is lower than both ordinary and extraordinary indices of the LC $\left(\mathrm{n}_{\mathrm{PCF}}<\mathrm{n}_{\mathrm{LCo}}<\mathrm{n}_{\mathrm{LCe}}\right)$. As mentioned before, it is a typical situation for the PLCFs based on silica glass and in such a case only PBG propagation is possible. As a experimental example of low index core PLCFs we present electrical tuning of the MCSU-1023 fiber (Fig. 2a) filled with a typical nematic liquid crystal 5 CB $\left(\mathrm{n}_{\mathrm{LCo}} \sim 1.52 ; \mathrm{n}_{\mathrm{LCe}} \sim 1.72\right)$. It is clear that for each voltage, light is guided by the PBG mechanism and only the modulation of attenuation is observed as a result of increased scattering of light during the reorientation of molecules (Fig. 3).

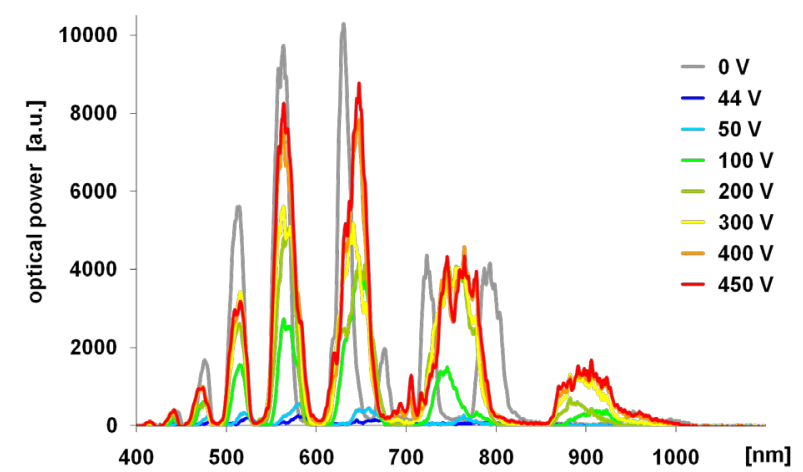

Fig. 3. Electric tuning of the PBGs in a low index core PLCF (MCSU-1023 filled with 5CB).

\section{Medium index-core PLCFs}

As medium index-core PLCFs we recognize the fibers based on solid-core PCFs made of glass, whose refractive index is below the extraordinary and above ordinary refractive index of the LC $\left(\mathrm{n}_{\mathrm{LCo}}<\mathrm{n}_{\mathrm{PCF}}<\mathrm{n}_{\mathrm{LCe}}\right)$. Numerical simulations proved that in such fibers guiding properties strongly depend on the alignment of LC molecules and polarization of light. In particular, if all molecules are parallel to the fiber axis (planar alignment, typically observed experimentally), both polarizations are index guided and the fiber is non-birefingent (assuming that hexagonal symmetry of the PCF is maintained). On the contrary, if all molecules are perpendicular to the fiber axis, one polarization is index-guided, since for orthogonal polarization only selected wavelengths can be guided by the PBG effect (Fig. 4).

Numerical simulations showed that in the case of transverse alignment of LC molecules in the medium index-core PLCFs also confinement losses strongly depend on polarization and the wavelength used (Fig. 5), since for planar alignment of the LC molecules a broadband and low-loss propagation is observed for both polarizations. This suggests that medium index-core
PLCFs seem to be good candidates to build effectively tunable polarizers and attenuators.

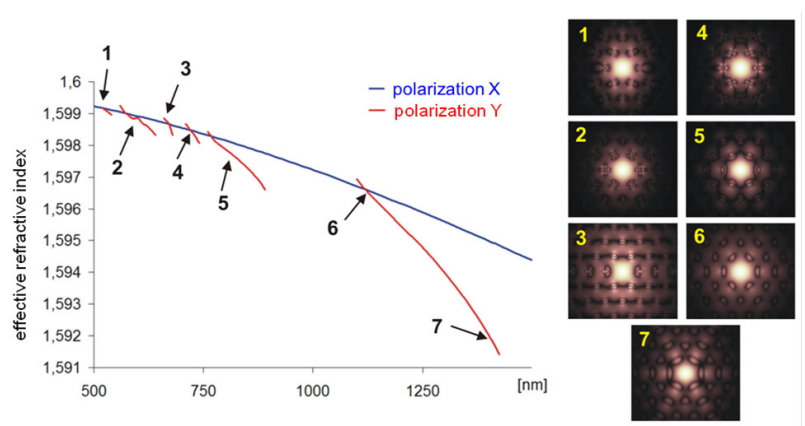

Fig. 4. Effective indices and PBG mode profiles calculated for IEMTPCF18 fiber filled with 5CB with the assumption of transverse alignment of LC molecules - simultaneous polarization dependent propagation of the PBG and index-guided modes.

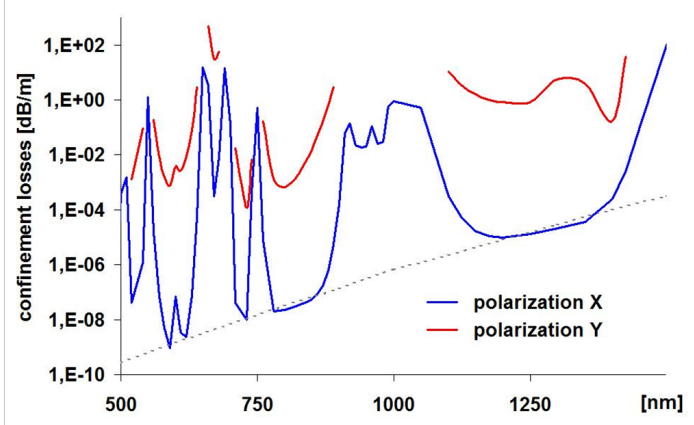

Fig. 5. Confinement losses calculated for modes presented in Fig. 4.

So far medium index-core PLCFs have been investigated only theoretically [i.e. 17, 18]. However, the guiding mechanism change observed in [7] can be discussed in terms of a change from a low index core PLCF into a medium index-core PLCF. Recently we have started experiments with PLCFs based on the IEMT PCF18 fiber, made of Schott-F2 glass (refractive index $\sim 1.62$ - Fig. 2b), so there is a wide range of LCs allowing for building medium index-core PLCFs. The results of those experiments will be reported soon.

\section{High index-core PLCFs}

As it could be expected, this class of PLCFs refers to the situation in which the refractive index of a solid-core host-PCF is higher than both refractive indices of the LC $\left(\mathrm{n}_{\mathrm{LCo}}<\mathrm{n}_{\mathrm{LCe}}<\mathrm{n}_{\mathrm{PCF}}\right)$. In this case the refractive index of the core is always higher than the effective index of the cladding (no matter how the LC molecules are aligned), so index-guiding is always possible in such fibers. It is a very interesting feature since index-guiding modes are strongly localized in the core and their confinement losses 
are smaller than those observed in the PBG modes. Moreover, it has been observed experimentally that attenuation of the PBG modes is much higher than theoretical minima defined by confinement losses, because PBG modes deeply penetrate the holes filled with an LC and light is strongly scattered. Typical losses of the PBG modes vary from a few to even a few tens of $\mathrm{dB} / \mathrm{cm}$ [2-14]. In the case of index-guided modes of high index core PLCFs light scattering by an LC is minimized due to the strong localization of light (according to numerical simulations usually more than $99 \%$ of the optical power is guided in the glass). It means that high index-core PLCFs allow for a significant reduction of attenuation. In [15] we have used the IEMT PCF-14 fiber made of PBG08 glass (Fig. 2c), whose refractive index is about $\sim 1.95$, and which allowed us to obtain attenuation at the level of 0.19 $\mathrm{dB} / \mathrm{cm}$ (to our best knowledge it has been the lowest attenuation so far reported in the class of liquid crystal waveguides).

As mentioned, in high index core PLCFs the alignment of LC molecules has no influence on the guiding mechanism. However, by changing the orientation of LC molecules it is possible to obtain effective broadband tuning of the birefringence and in [15] a continuous change of the birefringence from 0 to $2 \cdot 10^{-4}$ has been obtained. Such fibers can find application as tunable phase shifters, and recently a prototype of such a device has been assembled (Fig. 6).
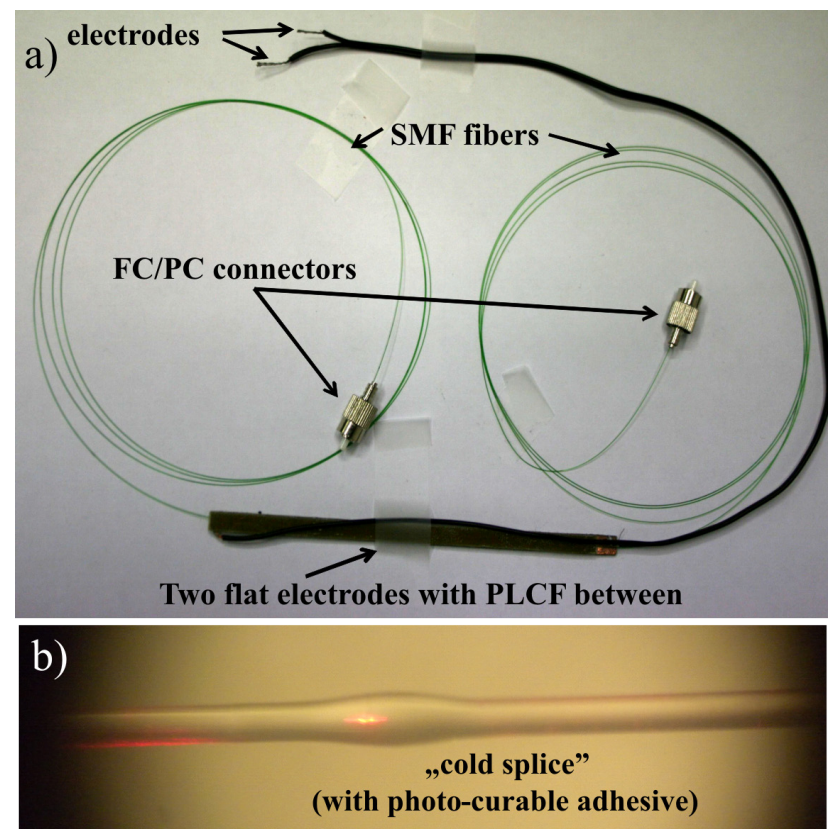

Fig. 6. Prototype of an electrically tunable phase shifter based on a high index core PLCF: a) general overview of the device; b) close up to the "cold splice" made with photo-curable adhesive.
To summarize, PLCFs can be divided into three classes with a different relation between the refractive indices of the guest LC and refractive index of the host PCF. PLCFs with a low-, medium- and high refractive index of the core exhibit different guiding properties, but it seems that each of the classes could find practical applications.

This work was supported by the Polish Ministry of Science and Education under the grant N517 554139 and partially by the "Master" Programme of the Polish Science Foundation.

\section{References}

[1] P. St.J. Russell, J. Light. Technol. 24, 4729-4749 (2006).

[2] T. Larsen, A. Bjarklev, D. Hermann, and J. Broeng, Opt. Exp. 11, 2589-2596 (2003).

[3] T. R. Wolinski, K. Szaniawska, K. Bondarczuk, P. Lesiak, A.W. Domanski, R. Dabrowski, E. Nowinowski-Kruszelnicki, J. Wojcik, Opto-Electronics Rev. 13(2), 59-64 (2005).

[4] J. Du, Y. Liu, Z. Wang, B. Zou, B. Liu, and X. Dong, Appl. Opt. 47, 5321-5324 (2008).

[5] M. Y. Jeon and J. H. Kim, Japanese Jpour. of Appl. Phys. 47(4), 2174 2175 (2008).

[6] M.W. Haakestad, T. T. Alkeskjold, M. D. Nielsen, L. Scolari, J. Riishede, H. E. Engan, and A. Bjarklev, IEEE Photon. Technol. Lett. 17, 819-821 (2005).

[7] T. R. Wolinski, K. Szaniawska, S. Ertman, P. Lesiak, A.W. Domanski, R. Dabrowski, E. Nowinowski-Kruszelnicki, and J. Wojcik, Meas. Sci. Technol. 17, 985-991 (2006).

[8] T. Alkeskjold, J. Lægsgaard, A. Bjarklev, D. Hermann, A. Anawati, J. Broeng, J. Li, and S.-T. Wu, Opt. Exp. 12, 5857-5871 (2004).

[9] V. K. Hsiao and C.-Y. Ko, Opt. Exp. 16, 12670-12676 (2008).

[10] L. Scolari, T. Alkeskjold, J. Riishede, A. Bjarklev, D. Hermann, A. Anawati, M. Nielsen, and P. Bassi, Opt. Exp. 13, 7483-7496 (2005).

[11] A. Lorenz, H.-S. Kitzerow, A. Schwuchow, J. Kobelke, and H. Bartelt, Opt. Exp. 16, 19375-19381 (2008).

[12] L. Scolari, S. Gauza, H. Xianyu, L. Zhai, L. Eskildsen, T. T. Alkeskjold, S.-T. Wu, and A. Bjarklev, Opt. Exp. 17, 3754-3764 (2009).

[13] T. T. Alkeskjold and A. Bjarklev, Opt. Lett. 32, 1707-1709 (2007).

[14] T. .R. Wolinski, S. Ertman, A. Czapla, P. Lesiak, K. Nowecka, A. W. Domanski, E. Nowinowski-Kruszelnicki, R. Dabrowski, and J. Wójcik Meas. Sci. Technol. 18, 3061-3069 (2007).

[15] S. Ertman, T. R. Wolinski, D. Pysz, R. Buczynski, E. NowinowskiKruszelnicki, and R. Dabrowski, Opt. Exp. 17, 19298-19310 (2009).

[16] D. Lorenc, M. Aranyosiova, R. Buczyński, R. Stępień, I. Bugar, A. Vincze, D. Velic, Appl. Phys. B: Lasers Opt. 93, 531-538 (2008).

[17] J. Sun and C. C. Chan, Opt. Lett. 32, 1989-1991 (2007).

[18] G. Ren, P. Shum, J. Hu, X. Yu, Y. Gong, J. Lightw. Technol. 26(22), 3650-3659 (2008). 